\title{
Symposium review: Embryo survival—A genomic perspective of the other side of fertility*
}

\section{H. Khatib† and N. Gross}

Department of Animal Sciences, University of Wisconsin, Madison 53706

\begin{abstract}
The majority of embryonic loss in cattle occurs within the first 3 to $4 \mathrm{wk}$ of pregnancy, and there are currently no accurate predictors of pregnancy outcome. Existing embryo quality assessment methods include morphological evaluation and embryo biopsy. These methods are not accurate and carry some health risks to the developing embryo, respectively. Therefore, there is need to identify noninvasive biomarkers such as microRNA that can predict embryo quality and pregnancy outcome. Furthermore, researchers need a better understanding of the dynamic interaction between the mother and the embryo. The transcriptome of the uterus shows plasticity that depends on the embryo type so that the expression level of some genes for in vivo embryos would be different from that of in vitroproduced embryos. Similarly, the embryonic transcriptome and epigenome change in response to different environmental factors such as stress, diet, disease, and physiological status of the mother. This embryo-mother crosstalk could be better understood by investigating the molecular signaling that occurs at different stages of embryonic development. Although transcriptomics is a useful tool to assess the roles of genes and pathways in embryo quality and maternal receptivity, it does not provide the exact functions of these genes, and it shows correlation rather than causality. Therefore, an indepth functional genomic analysis is needed for better understanding of the molecular mechanisms controlling embryo development. In this review, we discuss recent genomic technologies such as RNA interference, gapmer technology, and genome editing techniques used in humans and livestock to elucidate the molecular mechanisms of genes affecting embryo development.
\end{abstract}

Received June 20, 2018.

Accepted July 31, 2018.

*Presented as part of the Breeding and Genetics Symposium: Fertility - Filling the Gaps at the ADSA Annual Meeting, Knoxville, Tennessee, June 2018.

†Corresponding author: hkhatib@wisc.edu
Key words: noninvasive, embryo quality, RNA interference, gapmer technology, CRISPR

\section{GENETIC RELICS OF EMBRYO-MOTHER DIALOGUE ARE OPPORTUNITIES TO UNDERSTAND AND MANIPULATE COMPATIBILITY}

Approximately 30 to $70 \%$ of pregnancy losses occur in the first month of gestation (Wiltbank et al., 2016). Yet interactions between the mother and embryo at this stage remain difficult to interpret, and pregnancy loss is challenging to predict. Fundamentally, successful pregnancy requires a balance between a suitable maternal womb and a properly programmed embryo. On the one hand, the uterus helps to drive development and serves as a dynamic sensor of embryo quality. On the other hand, a successful embryo maintains basic tools for its own growth and adjusts to the uterine environment.

The bidirectional reaction of transcriptomes between the maternal endometrium and the embryo is a marker for embryo-mother crosstalk, and it could be used to determine a suitable maternal endometrium and a compatible and durable embryo. These interactions need to be better understood and investigated from both the embryo's and the mother's side of communication. Together, the maternal and embryonic transcriptomes can give a more complete picture of interactions occurring at their interface. Importantly, modifications to the genome, such as DNA methylation, should be considered for their roles in affecting RNA expression of embryos and maternal endometrium. Causative factors for differences in gene expression such as DNA methylation may lead to biomarkers and potential targets for infertility treatment.

\section{Transcriptional Plasticity of the Uterus}

The genomic functionality of the uterus is crucial to understand because the uterus serves as a vigorous sensor and driver of embryonic viability (Sandra et al., 2011). An underlying signature for changes occurring in the uterus may be seen in RNA profiles (Sandra et al., 
2011). These signatures could be useful tools for mitigation of infertility. One major factor that causes uterine remodeling is the presence of an embryo. Indeed, the transcriptome of a bovine uterus can differ based on the type of embryo it is exposed to, which has been recently reviewed (Sandra et al., 2011; Ulbrich et al., 2012). For instance, the endometrial transcriptome has differential expression when exposed to cloned versus standard in vitro-produced embryos (Bauersachs et al., 2009), and when exposed to in vitro-produced embryos versus in vivo-produced embryos (Mansouri-Attia et al., 2009). Furthermore, in vitro culture systems have revealed that endometrial cells may also transcriptionally respond to embryonic differences such as sex (Gross et al., 2017; Gómez et al., 2018).

Transcriptomic changes in the uterus during the preimplantation window are valuable to understand because embryo-mother compatibility during this stage is not well understood. However, it is currently hard to derive the direct biological meaning of the transcriptomic adjustments observed in the uterus. In practice, it is difficult to put the transcriptional changes into the context of infertility issues, because there is no standard in vivo model for inherent high versus low rates of early pregnancy loss in cattle or in any other species. This hurdle has recently been addressed with serial embryo transfer models to identify variation in cow fertility (Moraes et al., 2018). Yet, it is difficult to recapitulate natural conception differences in the absence of hormonal regimens and embryo transfer.

Another challenge is that the endometrial transcriptomes may differ inherently based on embryo status or on environmental factors. For instance, estrous cycle stage (Forde et al., 2009), circulating progesterone levels (Forde et al., 2011), and diet (Valour et al., 2013; Guggeri et al., 2018) are all correlated with changes in endometrial gene expression. Therefore, experiments must be carefully designed, and interpretation of endometrial transcriptomes must be performed cautiously. Current understanding of uterine response to embryo growth can be improved by evaluating the other side of the equation. Biologically, the plasticity of the uterus has another meaning: the embryo must also be flexible enough to react to the fluctuating status of the maternal endometrium to survive.

\section{Embryonic Transcriptional Responses to Maternal Status}

The embryo seems to maintain a level of transcriptional malleability that depends on exposure to external factors. One example is the sensitivity of embryos to changes in nutrient composition of their environment. For instance, male and female embryos can react dif- ferently to the composition of in vitro culture medium. Heras et al. (2016) compared transcriptional profiles of in vivo-generated blastocysts cultured in 2 different in vitro media conditions: with or without supplemental serum, a factor linked to large offspring syndrome. When both in vitro conditions were compared with in vivo-produced embryos, serum-exposed blastocysts had 5 times more differentially expressed genes than the serum-free cultured blastocysts. Additionally, when compared with in vivo conditions, under the same serum-containing conditions, male embryos had 3 times more differentially expressed genes than the female embryos. Conversely, no sexually dimorphic difference in the number of differentially expressed genes was seen for serum-free conditions (Heras et al., 2016). It is important to understand the consequences of various in vitro culture environments on the embryo, as well as the effects of the in vivo maternal environment on embryos. Embryos are also sensitive to changes in maternal diet. Peñagaricano et al. (2013) assessed the effects of feeding a minor amount of supplemental methionine to cows in the window from calving to embryo flushing. When embryos from cows fed methionine-supplemented diets were compared with embryos from cows fed a control diet, expression of genes related to embryonic development and immune response were found to be differentially regulated (Peñagaricano et al., 2013).

Long-term, dissimilarities in embryonic gene expression that result from either alteration in culture environment or maternal diet may have lifelong effects. Because changes in maternal gene expression occur in response to embryo development, it will be important to study the consequences of embryo-induced changes in uterine transcripts in the future. So far, the transcriptional profile of a welcoming uterine environment requires characterization. Likewise, understanding transcription from the perspective of the embryo's intrinsic quality can help to fill the gaps in knowledge about variation in uterine reactions to different types of embryos.

To have the best chance of successful pregnancy, the embryo must begin with a minimum level of inherent stamina. It seems this stamina is also represented in the transcriptome. For instance, pregnancy success can be correlated with transcriptional profiles of embryos (El-Sayed et al., 2006). Driver et al. (2012) compared transcriptional profiles of embryos that were either recovered through uterine flushing or created in vitro. Although stringent morphological standards were used for both groups of embryos, 45 genes were found to be differentially expressed between in vivo- and in vitroderived blastocysts. Further, altered genes were linked to cholesterol and sterol synthesis, system development, and cell differentiation (Driver et al., 2012). By dem- 
onstrating inherent differences between in vitro- and in vivo-produced embryos of similar morphology, the study highlights the importance of using additional biological parameters such as transcriptomic profiles in conjunction with morphological assessment.

In vitro-produced embryos also differ transcriptionally compared with embryos produced through somatic cell nuclear transfer (SCNT). Min et al. (2015) evaluated RNA sequencing (RNA-seq) data from individual blastocysts produced by either in vitro fertilization or SCNT to better understand incomplete programming of SCNT embryos. The study included both cumulus cell-derived and ear skin fibroblast-derived SCNT blastocysts in the analysis. Overall, transcriptional divergence was found between SCNT embryos and in vitro-produced embryos. Further, SCNT embryos produced using cumulus cells had more transcriptional similarities to in vitro embryos. Intriguingly, fibroblastderived SCNT embryos collectively showed lower expression of several genes linked to reprogramming (Min et al., 2015). In the future, the ties between embryonic transcriptional changes and uterine transcriptional adjustments relative to embryo-mother communication should be uncovered. Whether transcriptional differences in embryos produced in vitro or by SCNT directly cause differences in the maternal endometrium following transfer of these embryos has not been fully dissected.

\section{TAKING ADVANTAGE OF BIOLOGICAL PROCESSES: GENOMIC, EMBRYO-DERIVED, NONINVASIVE BIOMARKERS}

\section{Current Methods for Embryo Quality Assessment}

Current methods for embryo quality assessment do not fully utilize embryo-mother communication. Today's embryologist uses morphological assessment to select embryos. This assessment is qualitative and does not fully represent the internal genetics driving development of the embryo. For instance, in vivo- and in vitro-produced embryos can have similar morphology but different RNA expression (Driver et al., 2012). Further, in vitro-produced embryos have impaired success rates compared with in vivo embryos (Lonergan et al., 2006). Because the state of the genetic material of an embryo could indicate its developmental competence, there is a need to expand embryo assessment techniques beyond morphology.

A method that surveys the internal workings of an embryo is embryo biopsy. Biopsy is an avenue to assess transcriptomic differences that could lead to implantation success or failure (El-Sayed et al., 2006). However, the invasiveness of the procedure creates concern over the risk to embryo development (Scott et al., 2013). Ideally, methods to assess embryo quality require little risk to the viability of the developing embryo and are not technically challenging. Indeed, there are several promising noninvasive options to genetically assess embryo quality that should be further explored.

\section{Selecting Viable Embryos Using Noninvasive Genetic Markers}

The mother and the embryo must form a line of communication to establish and maintain pregnancy. Therefore, continued embryo quality inherently depends on proper embryo-mother crosstalk; thus, evaluation of embryo quality should include an assessment of signaling. Although interferon-tau (IFN- $\tau$ ) is recognized as a prominent embryo-derived signal for recognition of pregnancy in cattle (Ulbrich et al., 2012), a variety of other molecules have been identified as having roles in embryo-mother interactions. The signaling between the embryo and the mother is intricate and involves cooperation between various hormones, proteins, cytokines, chemokines, proteases, antiproteases, and growth factors. Interpretation of these complex exchanges is challenging, and profiling of so many different forms of communication can be technically difficult. Many of the instructions for production of various cell-signaling molecules are embedded in genomic sequences. Therefore, genomic activity may be a useful metric for evaluating embryo-mother communication. Fortunately, several types of nucleic acids have been recently discovered in embryo culture media that may help to predict the quality of embryo-mother interaction.

One possible noninvasive marker for embryo quality is an epigenetic nucleic acid called microRNA (miRNA); miRNA are noncoding RNA sequences that act to degrade or block translation of their target mRNA (Wahid et al., 2010), and they are found circulating in most bodily fluids, including bovine blood and follicular fluid (Noferesti et al., 2015). Interestingly, Kropp et al. (2014) found that miRNA are also secreted into culture medium by in vitro-produced embryos. Further, the profile of miRNA secreted into medium of individually cultured embryos that became blastocysts differed from that of embryos that degenerated (Kropp et al., 2014; Kropp and Khatib, 2015a). A functional role for miR-24, a miRNA upregulated in degenerate embryo medium, was demonstrated. A miR-24 mimic was added to the medium at the morula stage by Kropp and Khatib (2015a), leading to decreased embryo development to blastocyst stage and a reduction of its target transcript, CDKN1b. Additionally, alterations in miRNA expression in embryo culture medium have been correlated with implantation outcomes in humans 
(Rosenbluth et al., 2014). MicroRNA secretions into embryo culture medium were later shown to differ between male and female embryos (Gross et al., 2017). Three miRNA upregulated in the medium of female embryos all targeted the progesterone receptor transcript (PGR). Upon supplementation of each miRNA to endometrial cells in culture, maternal PGR expression was upregulated (Gross et al., 2017). Therefore, miRNA can act as noninvasive epigenetic reflections of embryo developmental competence, inherent chromosomal differences, and embryo-mother communication. MicroRNA are not yet used as a standard tool for clinical assessment of embryo quality, partly due to the complexity of roles a single miRNA can fill and to the lack of in vivo studies. However, the power of these tiny nucleic acids should not be overlooked, and their roles as useful biomarkers should be further pursued.

MicroRNA are stable in fluids because they are short, able to bind proteins (Wahid et al., 2010), and can be packaged within extracellular vesicles $(\mathbf{E V})$, which are membranous transport bodies that serve as shuttles for molecules such as miRNA, mRNA, DNA, and proteins (Kim et al., 2017). Bovine embryos can secrete EV to culture medium (Mellisho et al., 2017). They have been the focus of several recent studies because they have the ability to travel greater distances throughout the body. For instance, a recent study demonstrated that miRNA from adipose tissues were capable of regulating gene expression in liver tissues and that the transfer was dependent on EV (Thomou et al., 2017). In the future, EV profiles could be used in embryo quality assessment. Currently, characterization of the miRNA, mRNA, DNA, and protein potentially contained within these EV is lacking. However, it seems that an occasional passenger in $\mathrm{EV}, \mathrm{mRNA}$, is a potential candidate for assessing embryo quality; mRNA fragments have been identified in embryo medium and are associated with the quality of preimplantation development (Kropp and Khatib, 2015b). Although not fully characterized, mRNA related to pluripotency have been found contained within EV secreted to the medium of human embryos (Giacomini et al., 2017). With further profiling of these mRNA, it may be possible to use mRNA as markers to determine current transcriptional activity and competency of embryos.

Recently, DNA has also been identified in embryo culture medium (Stigliani et al., 2013; Galluzzi et al., 2015; Shamonki et al., 2016). Cell-free DNA assessment in embryo medium requires further development due to current difficulties with allele dropout rates (Galluzzi et al., 2015) and crossover of DNA from non-embryo sources into the culture medium (Hammond et al., 2017). However, the potential for DNA as a biomarker is vast. Genomic selection is already widely used in the dairy industry, so this poses an intriguing opportunity to develop assays for assessment of the genomic material in embryo culture medium to perform selection.

A universal challenge of studying nucleic acids secreted to embryo medium is the lack of consistency across culture systems. Variation across culture systems can cause challenges with reproducibility because the embryo can adjust based on its environment. Indeed, these inconsistencies likely reflect embryo quality as a result of a different environment. Although consistency in secretions across various culture systems should be characterized, differences should also be understood to improve culture system quality. Additionally, understanding the biological relevance of these nucleic acids can help uncover the fate of an embryo's development.

\section{Utilizing Noninvasive Markers as Tools to Improve Fertility}

Noninvasive genetic markers may have dual utility. Nucleic acids can aid in embryo-mother communication and they can be used to improve pregnancy outcomes. Recently, supplementation of maternal exosomes has been used to improve competency of SCNT embryos (Qiao et al., 2018). Additionally, parthenogenetic embryos cocultured with SCNT embryos improved development, and the exosomes produced by parthenogenetic embryos were taken up by SCNT embryos (Saadeldin et al., 2014). Currently, the complete catalog of EV contents is unknown and requires further characterization from both the maternal and embryonic sides of secretions. In the future, these secretions should be assessed for their direct links to pregnancy outcomes.

\section{MANIPULATING THE EMBRYONIC GENOME AND FUNCTIONAL GENOMICS APPROACHES}

\section{Embryonic Gene Expression}

Early developmental stages include the timing and progression through the first and subsequent cellular divisions, formation of necessary gap junctions, embryonic genome activation (EGA), compaction, blastocoel formation, differentiation of cell types, and secretion of embryonic gene products essential for maternal recognition. Transcriptomics can serve as an excellent tool to assess the roles of key genes and pathways involved in the different stages of embryo development.

Kues et al. (2008) studied the volume of embryonic mRNA transcripts through the different stages of development and reported that the greatest number of transcripts corresponded to the 2- to 4-cell stage, with fewer transcripts from the 4- to 8-cell stage. Those authors concluded that the observed lower number of 
4-to 8-cell stage transcripts was due to rapid degradation of maternal transcripts during maternal embryonic transition. Moreover, 350 genes were developmental stage-specific, with about 70 total genes more highly expressed in the 2-cell stage that were then downregulated in subsequent stages (Kues et al., 2008). Additionally, this study also classified the genes expressed at the 4- to 8-cell stage, which showed high metabolic and signaling activity, providing evidence of the dynamic changes occurring within the embryo leading up to EGA.

Although it is known that the embryo needs to undergo EGA for successful progression in development, the genetic mechanisms underlying development from the morula to blastocyst stage are even more unclear. Our laboratory has an established bovine embryo model for transcriptomic profiling to compare embryos that develop to the blastocyst stage with those that show abnormal and arrested development (deemed degenerate embryos) by Day 8 . Using a microarray analysis, 67 differentially expressed genes were found, 33 of which had greater than 2-fold differences in expression between the 2 embryo populations (Huang et al., 2010). Gene ontology analysis of the genes upregulated in the degenerate embryo population revealed that although many pathways were enriched, one of particular interest was the transforming growth factor (TGF)- $\beta$ pathway. Genes of this pathway have roles in cell division, mitosis, differentiation, and apoptosis.

The heat shock protein (HSP) genes are expressed early during development and are known to function as molecular chaperones to assist in protein folding, transport, and stability. Zhang et al. (2011) compared the expression of 17 HSP genes in blastocysts and degenerate embryos. All of these genes were differentially expressed, with expression differences ranging from 1.5 - to 7.6 -fold. The HSP40 genes were more highly expressed in degenerate embryos, whereas the HSP70 members were more highly expressed in blastocysts. Thus, expression and function of these genes may play an important role in mediating stress for successful development. Interestingly, the expression of these genes was found to be relevant to embryonic development when oocytes came from different sources (Pretheeban et al., 2009). In that study, parity and poor embryo quality were tested for their possible contributions to the lower pregnancy rates observed in mature cows compared with heifers. Semiquantitative PCR results revealed that HSP70 was more highly expressed in heifer embryos than in those from mature, multiparous cows. Collectively, the results from these studies indicate the extent of the influence that HSP genes have on the success of a developing embryo, with expression of HSP70 potentially underlying successful blastocyst development.

\section{Roles of Imprinted Genes in Early Embryonic Development}

One of the best-studied types of epigenetic modifications is DNA methylation, which alters gene expression (Reik and Dean, 2001). Methylation, which usually occurs at $\mathrm{CpG}$ sites within the DNA, can alter histone structure and, as a result, repress or activate gene expression. In imprinting, methylation causes only one parental allele to be expressed. Genes showing this phenomenon of parent-of-origin-specific, monoallelic expression are known as "imprinted" genes.

Imprinted genes are regulated by epigenetic modifications such as DNA methylation, histone modification, and chromatin remodeling (Kono, 2009), and they are known to play important roles in early embryonic development. For instance, mice lacking the maternally expressed gene Phlda2 show placental overgrowth (Frank et al., 2002), whereas overexpression of this gene was associated with delayed placental growth (Salas et al., 2004). When studying a bovine embryonic transcriptome, Huang et al. (2010) found that PHLDA2 was upregulated by about 8-fold in degenerate bovine embryos. Recently, Driver et al. (2013) reported that 5 imprinted genes (NDN, TSSC4, UBE3A, PEG3, and $M K R N 3)$ were upregulated in degenerate embryos and 4 imprinted genes (MAGEL2, NAP1L5, IGF2R, and $C D K N 1 C)$ were upregulated in healthy blastocysts. Functionally, the imprinted genes shown to have differential expression in the study of Driver et al. (2013) all play various roles that could influence early development. For example, the high expression of MKRN3 in degenerative versus blastocyst embryos suggests a possible lack of controlled RNA modifications causing cellular arrest and incomplete development. The protein encoded by MKRN3 putatively functions as a ribonucleoprotein and has been shown to be solely expressed after the maternal embryonic transition in bovine in vitro embryos, where maternal stores of RNA are degraded and the embryonic transcripts have gained control (Jong et al., 1999; Tveden-Nyborg et al., 2008). Overall, data from these studies provide evidence that proper expression of imprinted genes may be crucial for progression through embryonic developmental stages.

\section{Current Limitations in Embryo Transcriptomics}

Although transcriptomic studies in the preimplantation embryo have been insightful, they present a few key limitations. First, there is a lack of direct associa- 
tion between gene expression or function and embryo development. Currently, we have "snapshots" of what genes are active during the different stages without further evidence regarding which are critical to the embryo's development and survival. Second, these largescale studies have relied on microarray and RNA-seq expression technologies, which do not provide complete coverage of the genome, including alternative splicing and novel transcripts. Third, these high-throughput analyses are descriptive in nature and do not provide in-depth analysis of the functional roles of the differentially expressed genes in embryo development. Therefore, an in-depth functional analysis is needed for a better understanding of the molecular mechanisms controlling embryo development. In the next sections, we discuss some new technologies focused on functional genomics approaches used by our laboratory and by others to elucidate the roles of candidate genes in early embryonic development.

\section{RNA Interference}

RNA interference (RNAi) technology is utilized to assess gene function through the introduction of double-stranded RNA sequences of 9 to 29 nucleotides known as small interfering RNA (siRNA) into cells (Manrique et al., 2009). The siRNA is then unwound, and one RNA strand will bind an assembly of proteins to form the RNA-induced silencing complex (RISC). The RISC will choose its target based on the sequence complementarity between the siRNA and the mRNA target sequence, which in turn leads either to mRNA degradation or to translational repression (Manrique et al., 2009).

Several studies have used siRNA technology to silence specific bovine genes to determine their functions in embryo development (Nganvongpanit et al., 2006; Lee et al., 2009; Tesfaye et al., 2010). For example, microinjection of zygotes with Oct-4 sequence-specific double-stranded RNA resulted in a remarkable reduction in cleavage rates of zygotes, in protein synthesis of Oct-4, and in the number of cells reaching the blastocyst stage (Nganvongpanit et al., 2006). O'Meara et al. (2011) showed that the introduction of siRNA to bovine zygotes by either microinjection or transfection through Lipofectamine (Thermo Fisher Scientific, Waltham, MA) resulted in reduced expression of Ecadherin, a gene that has a role in embryo development. Driver et al. (2013) reported that the imprinted gene $C D K N 1 C$ was found to be highly expressed in normally developed blastocysts compared with degenerate embryos. Because of its involvement in the cell cycle, differentiation, and proliferation, its effects on neonatal lethality, and its association with embryo degeneration,
CDKN1C-siRNA was microinjected into 1-cell bovine embryos. Knockdown of $C D K N 1 C$ in the bovine zygotes by siRNA resulted in an approximate $46 \%$ reduction in blastocyst rate and a $60 \%$ reduction in $C D K N 1 C$ levels in Day 8 blastocysts compared with sham and control groups (Driver et al., 2013). These results led the authors to conclude that the differential expression observed between blastocyst and degenerates was causative, in part, of the embryos' degeneration.

Although siRNA technology provides an efficient and promising tool of gene silencing, off-target concerns have been raised by several research groups. Some of these off-target effects result from the homology of 6 to 7 nucleotides in the "seed region" of the siRNA sequence (Gavrilov and Saltzman, 2012). Another concern related to immunogenicity and toxicity is the possibility of activating interferon responses and cell death as a result of siRNA injection (Gavrilov and Saltzman, 2012).

\section{Gapmer Technology}

Gapmer technology is a new functional genomics tool based on the nucleotide base pairing between an antisense DNA oligonucleotide and a target mRNA. The gapmer oligonucleotide is composed of modified locked nucleic acids (LNA) containing a methylene bridge that connects the 2'-oxygen with the 4'-carbon of ribose that flank DNA monomers specific to a target mRNA of interest (Kurreck et al., 2002; Kauppinen et al., 2005). It has been shown that, in contrast to conventional antisense oligonucleotides, LNA oligonucleotides are biologically stable and have no detectable cell toxicity (Wahlestedt et al., 2000). Furthermore, the uptake of gapmers by cells in culture does not require transfection agents. Instead, gapmer uptake occurs via a process deemed "gymnosis" and has shown to efficiently target specific mRNA and knock down their expression (Stein et al., 2010; Soifer et al., 2012). Thus, a gene is silenced when the gapmer DNA monomers bind to the target mRNA and, upon the formation of the DNA:RNA heteroduplex, RNase $\mathrm{H}$ will cleave the RNA target strand (Kurreck et al., 2002; Kauppinen et al., 2005).

In a recent study, Kropp et al. (2017) evaluated differential gene expression between blastocysts generated from either high or low sire conception rate Holstein bulls; RNA sequencing analysis revealed 98 genes to be differentially expressed between the 2 embryo groups at a false discovery rate $<1 \%$. To further assess the roles of differentially expressed genes in embryonic development, the TFB2M gene was selected for knock down functional analysis using antisense oligonucleotide gapmer technology. The TFB2M gene plays a role in regulating metabolism at early stages of embryonic develop- 
ment through its role in transcription of mitochondrial (mt)DNA (Falkenberg et al., 2002). Supplementation to culture medium of zygotes of a gapmer oligonucleotide specific to TFB2M resulted in a significant reduction in TFB2M expression and reduced embryonic development to the blastocyst stage compared with nonsupplemented zygotes (Kropp et al., 2017).

Although gapmer technology has many advantages such as high stability and a lack of toxicity to injected cells, a major challenge of using this technology is the duration of the effects of silencing genes. A few days after zygote gapmer supplementation, the effects of the gapmer antisense vanish with each cell division cycle. Thus, there is a need to develop gene-silencing tools with longer lasting effects.

\section{Genome Editing Using the CRISPR/Cas9 System}

The CRISPR/Cas9 (clustered regularly interspaced short palindromic repeats/CRISPR-associated protein 9) system discovered in 2012 (Jinek et al., 2012) is a revolutionary genome editing technique in which a prokaryotic adaptive immune response system is used in a wide range of organisms to induce site-specific DNA double-strand breaks. These double-strand breaks can then be repaired either via the nonhomologous end joining DNA repair pathway (NHEJ) or the homology directed repair (HDR) pathway, using the cellular DNA repair mechanisms (Jinek et al., 2012). The use of the CRISPR/Cas9 system is relatively precise, inexpensive, and easy to implement; hundreds of CRISPR studies have been published since its discovery in 2012. However, these studies were limited to cell lines and animal models until the first use of genome editing in human embryos in 2015 by Liang et al. (2015). Those authors reported the use of CRISPR/Cas9 to repair mutations in the $\beta$-globin gene, $H B B$, which causes $\beta$-thalassemia, using human tripronuclear zygotes. Although successful in targeting and cleaving the $H B B$ gene, the use of Cas9 resulted in off-target cleavages, and the edited embryos were mosaic (Liang et al., 2015). The study of Liang et al. (2015) has ignited debates among the scientific community, including calls to halt these experiments because of ethical concerns and the possibility of health risks to the embryos (Lanphier et al., 2015).

Several genome-editing studies have been reported in livestock including pigs, sheep, and cattle. The first use of CRISPR technology in pigs was to knock down the $V W F$ gene to generate pigs with a phenotype similar to von Willebrand disease in humans (Hai et al., 2014). Microinjection into zygotes of Cas9 mRNA and single guide RNA (sgRNA) that targeted exon 5 of $V W F$ resulted in live piglets with a targeted mutation efficiency of $68 \%$. The authors reported little effect of genome editing procedures on early embryonic development, birth weight, or survival rate (Hai et al., 2014). In sheep, knock out of the myostatin gene in zygotes using CRISPR/Cas9 resulted in a stop codon and absence of myostatin protein (Crispo et al., 2015). Consequently, the knockout lambs were heavier than control lambs. In cattle, we reported that the imprinted gene PHLDA2 was highly expressed in degenerate in vitro-fertilized embryos compared with normally developed blastocyst embryos (Driver et al., 2013). Furthermore, microinjections of PHLDA2-specific siRNA to bovine zygotes resulted in a significant decrease in embryonic development to the blastocyst stage (Driver et al., 2013). Recently, we introduced CRISPR/Cas9 to zygotes to knock out PHLDA2. The CRISPR sgRNA were designed to guide Cas9 to delete exon 1 and part of exon 2 of the gene. The percentage of embryos attaining blastocyst stage by Day 8 was recorded. Table 1 shows blastocyst development in 3 different CRISPR microinjection experiments, including control embryos,

Table 1. Blastocyst development rates following clustered regularly interspaced short palindromic repeats $($ CRISPR $) \backslash$ Cas9 injections in bovine zygotes ${ }^{1}$

\begin{tabular}{lcccc}
\hline Treatment $^{1}$ & $\begin{array}{c}\text { Total } \\
\text { oocytes }\end{array}$ & $\begin{array}{c}\text { Unfertilized } \\
\text { oocytes }\end{array}$ & Blastocysts & $\begin{array}{c}\text { Blastocyst } \\
\text { development (\%) }\end{array}$ \\
\hline Control 1 & 116 & 26 & 30 & 33 \\
100 ng/ $\mu$ L CRISPR 1 & 141 & 48 & 41 & 44.1 \\
Control 2 & 145 & 36 & 47 & 43.11 \\
Sham 2 & 97 & 26 & 31 & 43.66 \\
100 ng/ $\mu$ L CRISPR 2 & 119 & 45 & 43 & 38.11 \\
Control 3 & 118 & 33 & 28 & 33.73 \\
Sham 3 & 112 & 29 & 28 & 47.06 \\
100 ng/ $/$ L CRISPR 3 & 52 & 18 & 16 & \\
\hline
\end{tabular}

${ }^{1}$ Bovine zygotes were injected with a CRISPR plasmid and a guide RNA plasmid that delete exon 1 and part of exon 2 of the PHLDA2 gene. A group of embryos that underwent mechanical puncturing without plasmid delivery (sham embryos) and non-treated embryos were used as controls. Blastocyst rates were recorded at day of development. Embryos injected with CRISPR plasmid showed higher development rates than controls in three independent experiments. 
embryos that underwent mechanical puncturing by the microinjection needle but no CRISPR delivery (to ensure that potential phenotypic observations were due to the CRISPR; i.e., sham), and CRISPR $\backslash$ Cas9 injections. Consistent with the previous results with siRNA injections (Driver et al., 2013), knock down of PHLDA2 caused increased embryonic development to the blastocyst stage.

\section{SUMMARY}

Current assessment methods of embryo quality and development are not accurate. Therefore, there is a need to develop noninvasive biomarkers such as miRNA for the prediction of successful pregnancy. Also, it is necessary to understand the molecular mechanisms controlling the dynamic interaction between the fetus and the mother. Recent technologies such as RNAi, gapmer, and CRISPR that have the ability to modify genomes of agricultural species are promising tools to enhance production and reproduction traits in livestock. However, given that most economically important traits are complex, the use of CRISPR faces several challenges, including discovery of causal variants for complex traits, social acceptance of the technology, off-target effects, mosaic outcomes in individual cells of the offspring, and the development of low-cost multiple edits in zygotes (Hickey et al., 2016). Interestingly, in a recent breakthrough study, researchers were able to repair a deletion in the paternal copy of $M Y B P C 3$ in human embryos, a gene involved in hypertrophic cardiomyopathy that causes sudden heart failure, using CRISPR technology (Ma et al., 2017). For the first time, the paternal allele was repaired using the wildtype maternal allele as a template without evidence of mosaicism or off-target effects (Ma et al., 2017).

\section{REFERENCES}

Bauersachs, S., S. E. Ulbrich, V. Zakhartchenko, M. Minten, M. Reichenbach, H.-D. Reichenbach, H. Blum, T. E. Spencer, and E. Wolf. 2009. The endometrium responds differently to cloned versus fertilized embryos. Proc. Natl. Acad. Sci. USA 106:5681-5686. https://doi.org/10.1073/pnas.0811841106.

Crispo, M., A. P. Mulet, L. Tesson, N. Barrera, F. Cuadro, P. C. Dos Santos-Neto, T. H. Nguyen, A. Crénéguy, L. Brusselle, I. Anegón, and A. Menchaca. 2015. Efficient generation of myostatin knockout sheep using CRISPR/Cas9 technology and microinjection into zygotes. PLoS One 10:e0136690. https://doi.org/10.1371/journal .pone.0136690.

Driver, A. M., W. Huang, J. Kropp, F. Peñagaricano, and H. Khatib. 2013. Knockdown of CDKN1C (p57kip2) and PHLDA2 results in developmental changes in bovine pre-implantation embryos. PLoS One 8. https://doi.org/10.1371/journal.pone.0069490.

Driver, A. M., F. Peñagaricano, W. Huang, K. R. Ahmad, K. S. Hackbart, M. C. Wiltbank, and H. Khatib. 2012. RNA-Seq analysis uncovers transcriptomic variations between morphologically similar in vivo- and in vitro-derived bovine blastocysts. BMC Genomics 13:118. https://doi.org/10.1186/1471-2164-13-118.
El-Sayed, A., M. Hoelker, F. Rings, D. Salilew, D. Jennen, E. Tholen, M.-A. Sirard, K. Schellander, and D. Tesfaye. 2006. Large-scale transcriptional analysis of bovine embryo biopsies in relation to pregnancy success after transfer to recipients. Physiol. Genomics 28:84-96. https://doi.org/10.1152/physiolgenomics.00111.2006.

Falkenberg, M., M. Gaspari, A. Rantanen, A. Trifunovic, N. G. Larsson, and C. M. Gustafsson. 2002. Mitochondrial transcription factors B1 and B2 activate transcription of human mtDNA. Nat. Genet. 31:289-294. https://doi.org/10.1038/ng909.

Forde, N., M. E. Beltman, G. B. Duffy, P. Duffy, J. P. Mehta, P. O'Gaora, J. F. Roche, P. Lonergan, and M. A. Crowe. 2011. Changes in the endometrial transcriptome during the bovine estrous cycle: Effect of low circulating progesterone and consequences for conceptus elongation. Biol. Reprod. 84:266-278. https://doi .org/10.1095/biolreprod.110.085910.

Forde, N., F. Carter, T. Fair, M. A. Crowe, A. C. O. Evans, T. E. Spencer, F. W. Bazer, R. McBride, M. P. Boland, P. O'Gaora, P. Lonergan, and J. F. Roche. 2009. Progesterone-regulated changes in endometrial gene expression contribute to advanced conceptus development in cattle. Biol. Reprod. 81:784-794. https://doi.org/ 10.1095/biolreprod.108.074336.

Frank, D., W. Fortino, L. Clark, R. Musalo, W. Wang, A. Saxena, C.-M. Li, W. Reik, T. Ludwig, and B. Tycko. 2002. Placental overgrowth in mice lacking the imprinted gene Ipl. Proc. Natl. Acad. Sci. USA 99:7490-7495. https://doi.org/10.1073/pnas.122039999.

Galluzzi, L., S. Palini, S. De Stefani, F. Andreoni, M. Primiterra, A. Diotallevi, C. Bulletti, and M. Magnani. 2015. Extracellular embryo genomic DNA and its potential for genotyping applications. Futur. Sci. OA 1. https://doi.org/10.4155/fso.15.62.

Gavrilov, K., and W. M. Saltzman. 2012. Therapeutic siRNA: Principles, challenges, and strategies. Yale J. Biol. Med. 85:187-200.

Giacomini, E., R. Vago, A. M. Sanchez, P. Podini, N. Zarovni, V. Murdica, R. Rizzo, D. Bortolotti, M. Candiani, and P. Viganò. 2017. Secretome of in vitro cultured human embryos contains extracellular vesicles that are uptaken by the maternal side. Sci. Rep. 7:5210. https://doi.org/10.1038/s41598-017-05549-w.

Gómez, E., M. J. Sánchez-Calabuig, D. Martin, S. Carrocera, A. Murillo, E. Correia-Alvarez, P. Herrero, N. Canela, A. Gutiérrez-Adán, S. Ulbrich, and M. Muñoz. 2018. In vitro cultured bovine endometrial cells recognize embryonic sex. Theriogenology 108:176-184. https://doi.org/10.1016/j.theriogenology.2017.11.038.

Gross, N., J. Kropp, and H. Khatib. 2017. Sexual dimorphism of miRNAs secreted by bovine in vitro-produced embryos. Front. Genet. 8:39. https://doi.org/10.3389/fgene.2017.00039.

Guggeri, D., A. Meikle, M. Carriquiry, I. De Barbieri, F. Montossi, and C. Viñoles. 2018. Long-term effect of early nutrition on endocrine parameters and liver and endometrial gene expression of the members of the somatotrophic axis in Hereford heifers. Reprod. Domest. Anim. 53:930-936. https://doi.org/10.1111/rda.13190.

Hai, T., F. Teng, R. Guo, W. Li, and Q. Zhou. 2014. One-step generation of knockout pigs by zygote injection of CRISPR/Cas system. Cell Res. 24:372-375. https://doi.org/10.1038/cr.2014.11.

Hammond, E. R., B. C. McGillivray, S. M. Wicker, J. C. Peek, A. N. Shelling, P. Stone, L. W. Chamley, and L. M. Cree. 2017. Characterizing nuclear and mitochondrial DNA in spent embryo culture media: genetic contamination identified. Fertil. Steril. 107:220228.e5. https://doi.org/10.1016/j.fertnstert.2016.10.015.

Heras, S., D. I. M. De Coninck, M. Van Poucke, K. Goossens, O. Bogado Pascottini, F. Van Nieuwerburgh, D. Deforce, P. De Sutter, J. L. M. R. Leroy, A. Gutierrez-Adan, L. Peelman, and A. Van Soom. 2016. Suboptimal culture conditions induce more deviations in gene expression in male than female bovine blastocysts. BMC Genomics 17:72. https://doi.org/10.1186/s12864-016-2393-z.

Hickey, J. M., C. Bruce, A. Whitelaw, and G. Gorjanc. 2016. Promotion of alleles by genome editing in livestock breeding programmes. J. Anim. Breed. Genet. 133:83-84. https://doi.org/10.1111/jbg .12206 .

Huang, W., B. S. Yandell, and H. Khatib. 2010. Transcriptomic profiling of bovine IVF embryos revealed candidate genes and pathways involved in early embryonic development. BMC Genomics 11:23. https://doi.org/10.1186/1471-2164-11-23. 
Jinek, M., K. Chylinski, I. Fonfara, M. Hauer, J.A. Doudna, and E. Charpentier. 2012. A programmable dual-RNA-guided DNA endonuclease in adaptive bacterial immunity. Science 337:816-821. https://doi.org/10.1126/science.1225829.

Jong, M. T. C., A. H. Carey, K. A. Caldwell, M. H. Lau, M. A. Handel, D. J. Driscoll, C. L. Stewart, E. M. Rinchik, and R. D. Nicholls. 1999. Imprinting of a RING zinc-finger encoding gene in the mouse chromosome region homologous to the Prader-Willi syndrome genetic region. Hum. Mol. Genet. 8:795-803. https://doi .org/10.1093/hmg/8.5.795.

Kauppinen, S., B. Vester, and J. Wengel. 2005. Locked nucleic acid (LNA): High affinity targeting of RNA for diagnostics and therapeutics. Drug Discov. Today. Technol. 2:287-290. https://doi.org/ 10.1016/j.ddtec.2005.08.012.

Kim, K. M., K. Abdelmohsen, M. Mustapic, D. Kapogiannis, and M. Gorospe. 2017. RNA in extracellular vesicles. Wiley Interdiscip. Rev. RNA 8:1-14. https://doi.org/10.1002/wrna.1413.

Kono, T. 2009. Genetic modification for bimaternal embryo development. Reprod. Fertil. Dev. 21:31-36. https://doi.org/10.1071/ RD08213.

Kropp, J., J. A. Carrillo, H. Namous, A. Daniels, S. M. Salih, J. Song, and H. Khatib. 2017. Male fertility status is associated with DNA methylation signatures in sperm and transcriptomic profiles of bovine preimplantation embryos. BMC Genomics 18:280. https://doi .org/10.1186/s12864-017-3673-y.

Kropp, J., and H. Khatib. 2015a. Characterization of microRNA in bovine in vitro culture media associated with embryo quality and development. J. Dairy Sci. 98:6552-6563. https://doi.org/10.3168/ jds.2015-9510.

Kropp, J., and H. Khatib. 2015b. mRNA fragments in in vitro culture media are associated with bovine preimplantation embryonic development. Front. Genet. 6:273. https://doi.org/10.3389/fgene .2015.00273.

Kropp, J., S. M. Salih, and H. Khatib. 2014. Expression of microRNAs in bovine and human pre-implantation embryo culture media. Front. Genet. 5:91. https://doi.org/10.3389/fgene.2014.00091.

Kues, W. A., S. Sudheer, D. Herrmann, J. W. Carnwath, V. Havlicek, U. Besenfelder, H. Lenrach, J. Adjaye, and H. Niemann. 2008. Genome-wide expression profiling reveals distinct clusters of transcriptional regulation during bovine preinplantation development in vivo. Proc. Natl. Acad. Sci. USA 105:19768-19773. https://doi .org/10.1073/pnas.0805616105.

Kurreck, J., E. Wyszko, C. Gillen, and V. Erdmann. 2002. Design of antisense oligonucleotides stabilized by locked nucleic acids. Nucleic Acids Res. 30:1911-1918. https://doi.org/10.1093/nar/30 .9 .1911

Lanphier, E., F. Urnov, S. E. Haecker, M. Werner, and J. Smolenski. 2015. Don't edit the human germ line. Nature 519:410-411. https: //doi.org/10.1038/519410a.

Lee, K. B., A. Bettegowda, G. Wee, J. J. Ireland, and G. W. Smith. 2009. Molecular determinants of oocyte competence: Potential functional role for maternal (oocyte-derived) follistatin in promoting bovine early embryogenesis. Endocrinology 150:2463-2471. https://doi.org/10.1210/en.2008-1574.

Liang, P., Y. Xu, X. Zhang, C. Ding, R. Huang, Z. Zhang, J. Lv, X. Xie, Y. Chen, Y. Li, Y. Sun, Y. Bai, Z. Songyang, W. Ma, C. Zhou, and J. Huang. 2015. CRISPR/Cas9-mediated gene editing in human tripronuclear zygotes. Protein Cell 6:363-372. https:// doi.org/10.1007/s13238-015-0153-5.

Lonergan, P., T. Fair, D. Corcoran, and A. C. O. Evans. 2006. Effect of culture environment on gene expression and developmental characteristics in IVF-derived embryos. Theriogenology 65:137152. https://doi.org/10.1016/j.theriogenology.2005.09.028.

Ma, H., N. Marti-Gutierrez, S. W. Park, J. Wu, Y. Lee, K. Suzuki, A. Koski, D. Ji, T. Hayama, R. Ahmed, H. Darby, C. Van Dyken, Y. Li, E. Kang, A. R. Park, D. Kim, S. T. Kim, J. Gong, Y. Gu, X. Xu, D. Battaglia, S. A. Krieg, D. M. Lee, D. H. Wu, D. P. Wolf, S. B. Heitner, J. C. I. Belmonte, P. Amato, J. S. Kim, S. Kaul, and S. Mitalipov. 2017. Correction of a pathogenic gene mutation in human embryos. Nature 548:413-419. https://doi.org/10.1038/ nature23305.
Manrique, C., V. Compan, C. Rosselet, and S. G. D. Duflo. 2009 Specific knock-down of GAD67 in the striatum using naked small interfering RNAs. J. Biotechnol. 142:185-192. https://doi.org/10 .1016/j.jbiotec.2009.05.009

Mansouri-Attia, N., O. Sandra, J. Aubert, S. Degrelle, R. E. Everts, C. Giraud-Delville, Y. Heyman, L. Galio, I. Hue, X. Yang, X. C. Tian, H. A. Lewin, and J.-P. Renard. 2009. Endometrium as an early sensor of in vitro embryo manipulation technologies. Proc. Natl. Acad. Sci. USA 106:5687-5692. https://doi.org/10.1073/ pnas.0812722106.

Mellisho, E. A., A. E. Velásquez, M. J. Nuñez, J. G. Cabezas, J. A. Cueto, C. Fader, F. O. Castro, and L. Rodríguez-Álvarez. 2017. Identification and characteristics of extracellular vesicles from bovine blastocysts produced in vitro. PLoS One 12:e0178306. https: //doi.org/10.1371/journal.pone.0178306.

Min, B., S. Cho, J. S. Park, Y.-G. Lee, N. Kim, and Y.-K. Kang. 2015. Transcriptomic features of bovine blastocysts derived by somatic cell nuclear transfer. G3 (Bethesda) 5:2527-2538. https://doi.org/ 10.1534/g3.115.020016.

Moraes, J. G. N., S. K. Behura, T. W. Geary, P. J. Hansen, H. L. Neibergs, and T. E. Spencer. 2018. Uterine influences on conceptus development in fertility-classified animals. Proc. Natl. Acad. Sci. USA 115:E1749-E1758. https://doi.org/10.1073/pnas.1721191115. Nganvongpanit, K., H. Müller, F. Rings, M. Hoelker, D. Jennen, E. Tholen, V. Havlicek, U. Besenfelder, K. Schellander, and D. Tesfaye. 2006. Selective degradation of maternal and embryonic transcripts in in vitro produced bovine oocytes and embryos using sequence specific double-stranded RNA. Reproduction 131:861-874. https://doi.org/10.1530/rep.1.01040.

Noferesti, S. S., M. M. H. Sohel, M. Hoelker, D. Salilew-Wondim, E. Tholen, C. Looft, F. Rings, C. Neuhoff, K. Schellander, and D. Tesfaye. 2015. Controlled ovarian hyperstimulation induced changes in the expression of circulatory miRNA in bovine follicular fluid and blood plasma. J. Ovarian Res. 8:81. https://doi.org/10.1186/ s13048-015-0208-5.

O'Meara, C. M., J. D. Murray, S. Mamo, E. Gallagher, J. Roche, and P. Lonergan. 2011. Gene silencing in bovine zygotes: siRNA transfection versus microinjection. Reprod. Fertil. Dev. 23:534-543. https://doi.org/10.1071/RD10175.

Peñagaricano, F., A. H. Souza, P. D. Carvalho, A. M. Driver, R. Gambra, J. Kropp, K. S. Hackbart, D. Luchini, R. D. Shaver, M. C. Wiltbank, and H. Khatib. 2013. Effect of maternal methionine supplementation on the transcriptome of bovine preimplantation embryos. PLoS One 8:e72302. https://doi.org/10.1371/journal .pone.0072302.

Pretheeban, T., M. Gordon, R. Singh, R. Perera, and R. Rajamahendran. 2009. Differential mRNA expression in in vivo produced pre-implantation embryos of dairy heifers and mature cows. Mol. Reprod. Dev. 76:1165-1172. https://doi.org/10.1002/mrd.21084.

Qiao, F., H. Ge, X. Ma, Y. Zhang, Z. Zuo, M. Wang, Y. Zhang, and Y. Wang. 2018. Bovine uterus-derived exosomes improve developmental competence of somatic cell nuclear transfer embryos. Theriogenology 114:199-205. https://doi.org/10.1016/j.theriogenology 2018.03.027.

Reik, W., and W. Dean. 2001. DNA methylation and mammalian epigenetics. Electrophoresis 22:2838-2843.

Rosenbluth, E. M., D. N. Shelton, L. M. Wells, A. E. T. Sparks, and B. J. Van Voorhis. 2014. Human embryos secrete microRNAs into culture media-A potential biomarker for implantation. Fertil. Steril. 101:1493-1500. https://doi.org/10.1016/j.fertnstert.2014.01 .058 .

Saadeldin, I. M., S. J. Kim, Y. Bin Choi, and B. C. Lee. 2014. Improvement of cloned embryos development by co-culturing with parthenotes: A possible role of exosomes/microvesicles for embryos paracrine communication. Cell. Reprogram. 16:223-234. https:// doi.org/10.1089/cell.2014.0003.

Salas, M., R. John, A. Saxena, S. Barton, D. Frank, G. Fitzpatrick, M. J. Higgins, and B. Tycko. 2004. Placental growth retardation due to loss of imprinting of Phlda2. Mech. Dev. 121:1199-1210. https: //doi.org/10.1016/j.mod.2004.05.017. 
Sandra, O., N. Mansouri-Attia, and R. G. Lea. 2011. Novel aspects of endometrial function: A biological sensor of embryo quality and driver of pregnancy success. Reprod. Fertil. Dev. 24:68-79. https:/ /doi.org/10.1071/RD11908.

Scott, R. T., K. M. Upham, E. J. Forman, T. Zhao, and N. R. Treff. 2013. Cleavage-stage biopsy significantly impairs human embryonic implantation potential while blastocyst biopsy does not: A randomized and paired clinical trial. Fertil. Steril. 100:624-630. https://doi.org/10.1016/j.fertnstert.2013.04.039.

Shamonki, M. I., H. Jin, Z. Haimowitz, and L. Liu. 2016. Proof of concept: Preimplantation genetic screening without embryo biopsy through analysis of cell-free DNA in spent embryo culture media. Fertil. Steril. 106:1312-1318. https://doi.org/10.1016/j.fertnstert 2016.07.1112.

Soifer, H. S., T. Koch, J. Lai, B. Hansen, A. Hoeg, H. Oerum, and C. A. Stein. 2012. Silencing of gene expression by gymnotic delivery of antisense oligonucleotides. Pages 333-346 in Functional Genomics: Methods and Protocols. M. Kaufmann and C. Klinger, ed. Humana Press/Springer, New York, NY. https://doi.org/10.1007/ 978-1-61779-424-7.

Stein, C. A., J. B. Hansen, J. Lai, S. J. Wu, A. Voskresenskiy, A. Høg, J. Worm, M. Hedtjärn, N. Souleimanian, P. Miller, H. S. Soifer, D. Castanotto, L. Benimetskaya, H. Ørum, and T. Koch. 2010. Efficient gene silencing by delivery of locked nucleic acid antisense oligonucleotides, unassisted by transfection reagents. Nucleic Acids Res. 38:e3. https://doi.org/10.1093/nar/gkp841.

Stigliani, S., P. Anserini, P. L. Venturini, and P. Scaruffi. 2013. Mitochondrial DNA content in embryo culture medium is significantly associated with human embryo fragmentation. Hum. Reprod. 28:2652-2660. https://doi.org/10.1093/humrep/det314.

Tesfaye, D., A. Regassa, F. Rings, N. Ghanem, C. Phatsara, E. Tholen, R. Herwig, C. Un, K. Schellander, and M. Hoelker. 2010. Suppression of the transcription factor MSX1 gene delays bovine preimplantation embryo development in vitro. Reproduction 139:857870. https://doi.org/10.1530/REP-09-0312.

Thomou, T., M. A. Mori, J. M. Dreyfuss, M. Konishi, M. Sakaguchi, C. Wolfrum, T. N. Rao, J. N. Winnay, R. Garcia-Martin, S. K.
Grinspoon, P. Gorden, and C. R. Kahn. 2017. Adipose-derived circulating miRNAs regulate gene expression in other tissues. Nature 542:450-455. https://doi.org/10.1038/nature21365.

Tveden-Nyborg, P. Y., N. I. Alexopoulos, M. A. Cooney, A. J. French, R. T. Tecirlioglu, M. K. Holland, P. D. Thomsen, and N. T. D'Cruz. 2008. Analysis of the expression of putatively imprinted genes in bovine peri-implantation embryos. Theriogenology 70:1119-1128. https://doi.org/10.1016/j.theriogenology.2008.06.033.

Ulbrich, S. E., E. Wolf, and S. Bauersachs. 2012. Hosting the preimplantation embryo: Potentials and limitations of different approaches for analysing embryo-endometrium interactions in cattle. Reprod. Fertil. Dev. 25:62-70. https://doi.org/10.1071/RD12279.

Valour, D., I. Hue, S. A. Degrelle, S. Déjean, G. Marot, O. Dubois, G. Germain, P. Humblot, A. Ponter, G. Charpigny, and B. Grimard. 2013. Pre- and post-partum mild underfeeding influences gene expression in the reproductive tract of cyclic dairy cows. Reprod. Domest. Anim. 48:484-499. https://doi.org/10.1111/rda.12113.

Wahid, F., A. Shehzad, T. Khan, and Y. Y. Kim. 2010. MicroRNAs: Synthesis, mechanism, function, and recent clinical trials. Biochim. Biophys. Acta 1803:1231-1243. https://doi.org/10.1016/j.bbamcr .2010.06.013.

Wahlestedt, C., P. Salmi, L. Good, J. Kela, T. Johnsson, T. Hokfelt, C. Broberger, F. Porreca, J. Lai, K. Ren, M. Ossipov, A. Koshkin, N. Jakobsen, J. Skouv, H. Oerum, M. H. Jacobsen, and J. Wengel. 2000. Potent and nontoxic antisense oligonucleotides containing locked nucleic acids. Proc. Natl. Acad. Sci. USA 97:5633-5638. https://doi.org/10.1073/pnas.97.10.5633.

Wiltbank, M. C., G. M. Baez, A. Garcia-Guerra, M. Z. Toledo, P. L. J. Monteiro, L. F. Melo, J. C. Ochoa, J. E. P. Santos, and R. Sartori. 2016. Pivotal periods for pregnancy loss during the first trimester of gestation in lactating dairy cows. Theriogenology 86:239-253. https://doi.org/10.1016/j.theriogenology.2016.04.037.

Zhang, B., F. Peñagaricano, A. Driver, H. Chen, and H. Khatib. 2011. Differential expression of heat shock protein genes and their splice variants in bovine preimplantation embryos. J. Dairy Sci. 94:41744182. https://doi.org/10.3168/jds.2010-4137. 